\title{
Detection of evolving viruses
}

\author{
Yih Lee and John Yin* \\ Biotechnology and Biochemical Engineering Program, Thayer School of Engineering, Dartmouth College, Hanover, NH 03755-8000. \\ ${ }^{*}$ Corresponding author (e-mail: jyin@dartmouth.edu).
}

Received 26 December 1995; accepted 5 February 1996.

\begin{abstract}
The spread of viruses on a homogeneous lawn of receptive hosts provides an opportunity to detect the dynamics of their evolution. We have previously found that when repeated virus passages are confined to the expanding perimeter of a growing plaque, the appearance and outgrowth of genetically diverse strains (all descended from the same parent strain) can be traced along different radii of the plaque. As a plaque grows, the random mutation and selection of new fast-growing strains reduce the roundness or circularity of the growing plaque. Here we have quantified such changes in growing plaques of bacteriophage $\mathrm{T7}$ using a digital imaging system. We find that $\mathrm{T7}$ populations not adapted for fast growth exhibit a broader diversity of growth rates than populations adapted for fast growth. These results provide a foundation for understanding how viruses exploit mutation and selection processes to persist in nature.
\end{abstract}

Keywords: natural selection, fitness, population heterogeneity, bacteriophage replication, plaque morphology, image analysis

Viruses owe their persistence in nature to the heterogeneity of their populations, which enables them to evade antiviral agents and strategies $^{1-10}$. Several studies have shown that opportunities for the emergence of new viral strains arise as viruses are passaged between susceptible hosts in both natural ${ }^{11-14}$ and laboratory ${ }^{15-19}$ environments. Measuring the heterogeneity of a population sampled from a natural or laboratory environment requires that a large number of subpopulations be cloned and characterized by genotype or phenotype. Such measurements are time-consuming and labor-intensive, and they become still more involved when one seeks to observe the dynamics of such mixed populations. For laboratory investigations these challenges can be further complicated by impure viral stocks containing scarcely detectable, but significant, subpopulations ${ }^{20-22}$.

In designing experimental systems to characterize the evolution of viruses, we have acquired insights from recent studies on spatially resolved replication and evolution of RNA templates ${ }^{23,24}$. $\mathrm{McC}$ askill and coworkers followed the in vitro replication of RNA templates along thin capillaries containing an RNA-dependent RNA polymerase and nucleotide triphosphate precursors. This system provided a constant environment for RNA replication over a thousand generations as a traveling concentration wave. RNA adaptation to faster replication rates could be directly detected as increases in the front velocity ${ }^{23}$. Furthermore, carrying out the reaction in a massively parallel configuration enabled them to observe and quantify the stochastic nature of the mutational events ${ }^{24}$. We have previously reported that the emergence of viral mutants in growing plaques, like the appearance of RNA mutants in the capillaries, can be spatially resolved at the level of both phenotype and genotype ${ }^{25}$. Such high-fitness mutants appear at random. We expect their appearance and outgrowth to be reflected at any point in time in the overall plaque morphology.

In this study we examine the dynamics of viral populations by visualizing their propagation in a growing plaque. Since a plaque is initiated by the infection of a single host cell by a single virion, the stock culture is homogeneous by definition. As favorable mutant strains emerge along the expanding plaque perimeter, their faster rates along different radii introduce asymmetries into

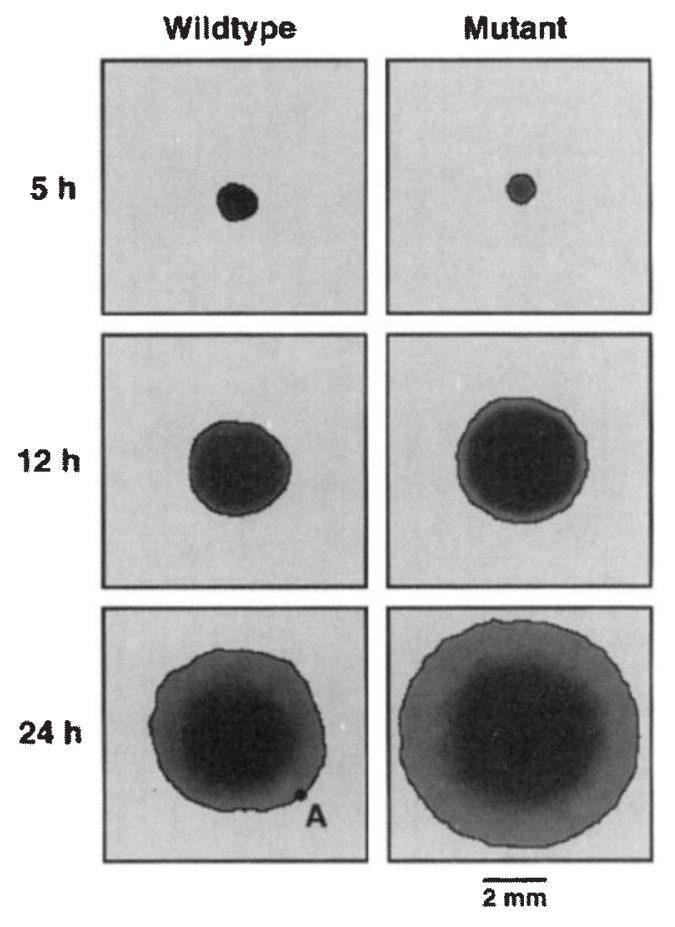

Figure 1. Plaque growth of bacteriophage T7: wildtype (left panels) and a fast-growing mutant (right panels). The mutant was isolated from a stab taken at point $A$ of the wildtype descendants, and then diluted and plated out as described in the Experimental protocol. 


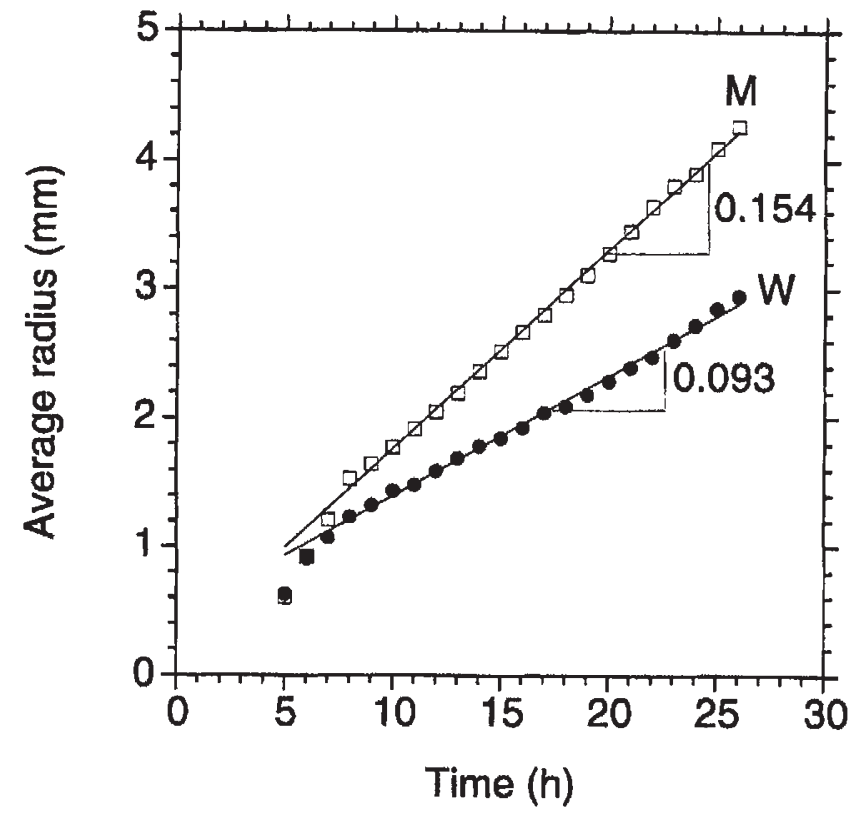

Figure 2. Growth rates of plaques initiated by wildtype $(W)$ and mutant $(\mathrm{M})$ virions. The average radial growth rates for the wildtype $(0.093 \mathrm{~mm} / \mathrm{h})$ and the mutant $(0.154 \mathrm{~mm} / \mathrm{h})$ were obtained by linear regression.

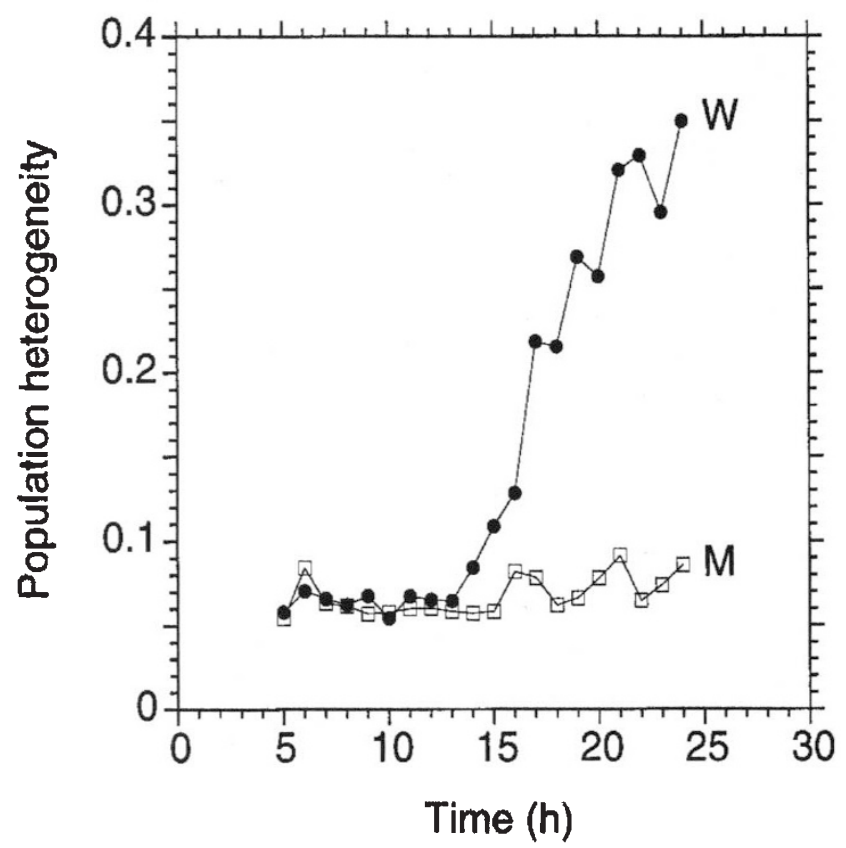

Figure 4. Dynamics of populations descended from a single wildtype (W) or fast-growing mutant (M) virion. The population heterogeneity has a minimum value of zero for a perfectly circular plaque.

the plaque shape. Bacteriophage T7 serves as our model system to initiate studies of emergent heterogeneity. During the $10^{8}$ - to $10^{10}$-fold amplification ${ }^{26}$ of a single T7 virion in a growing plaque, a variety of mutant strains emerge along different radii of the plaque ${ }^{25}$. They are genetically different than the wildtype and replicate more rapidly. In this study, we first demonstrate the feasibility of imaging techniques for monitoring and quantifying the

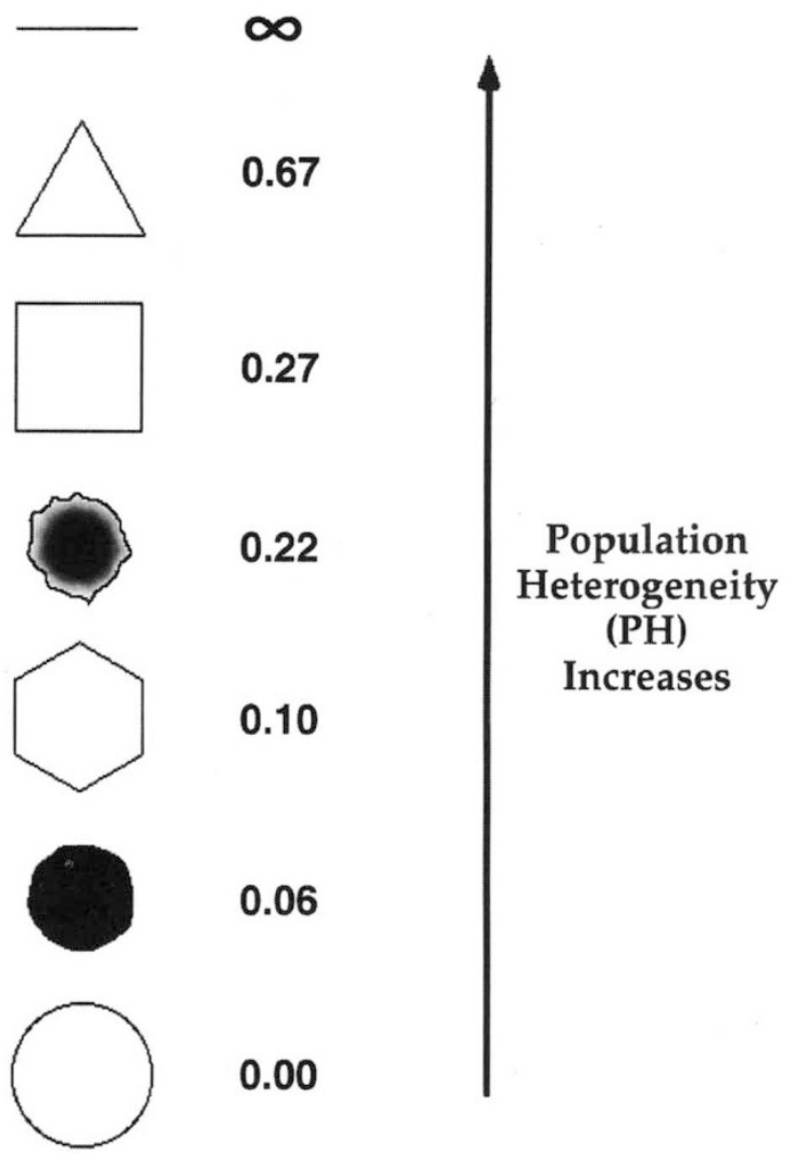

Figure 3. Population heterogeneity $(P H)$ for various shapes based on area and perimeter.

expansion of a plaque. We distinguish the mutant from the wildtype by the difference of average growth rates of plaques. Finally, we identify the emergence of mutating viruses by comparing the plaque morphology based on a simple geometric measure of circularity.

\section{Results and discussion}

Three images from the growth of a plaque initiated by wildtype phage T7 are shown in Figure 1 (left panels). After 8 hours the average radius of the plaque increases linearly with time (curve W, Fig. 2). The slope depends on the rate of viral replication within its host as well as the diffusion coefficient of the virus ${ }^{27}$. Images of a fast-growing mutant stab-isolated from point $\mathrm{A}$ (Fig. 1), a slightly bulged region of the plaque, are shown in Figure 1 (right panels). We find that the mutant (curve $M$ ) grew more than $60 \%$ faster than its wildtype precursor (curve W, Fig. 2).

Figure 3 illustrates the values of population heterogeneity $(P H)$ for several geometric shapes including two actual plaque images. If all virus populations in the spreading perimeter of a plaque propagate at the same rate, the plaque should maintain a circular shape as it grows; its $\mathrm{PH}$ should remain at zero as the plaque expands. However, as faster-growing mutants arise randomly at different positions along the plaque perimeter, they cause deviations from the circular shape. We find that $P H$ increases with time as the wild-type-initiated plaque grows; the populations become more heterogeneous (Fig. 4). However, the plaque that is initiated by a fast-growing mutant exhibits a low $\mathrm{PH}$ that remains constant over time; the population is homoge- 
neous with respect to plaque growth (Fig. 4, curve M). These results suggest that a wildtype strain which has not been adapted for efficient plaque growth will have a higher probability of generating higher fitness (e.g., faster propagating) mutants than a mutant strain that has already been selected for high fitness, as one might expect. We anticipate that studies incorporating mutagens and agents to perturb the virus-host interactions during plaque growth will provide further insights into the dynamics of evolving viral populations.

\section{Experimental protocol}

Plaque growth in single layer agar. Established procedures were employed in the preparation, preservation, and concentration determinations of the bacteriophage and bacteria ${ }^{28-30}$. Phage and host strains were provided by F. William Studier. To diminish the nutrient and phage fluxes in the vertical direction, plaques were grown in single-layer agar as described ${ }^{26}$, with minor modifications. Host $E$. coli was grown at $37^{\circ} \mathrm{C}$ in $10 \mathrm{~g} / \mathrm{L}$ nutrient broth (DIFCO, Detroit, MI) overnight to a concentration of about $2 \times 10^{8} / \mathrm{ml}$. Diluted phage T7 and $200 \mu \mathrm{l}$ cell culture were added to $12 \mathrm{ml}$ of $0.7 \%$ nutrient agar $(10 \mathrm{~g} / \mathrm{L}$ nutrient broth with $7 \mathrm{~g} / \mathrm{L}$ BactoAgar, DIFCO) at $55^{\circ} \mathrm{C}$. The entire mixture was gently vortexed and poured into an $8.5-\mathrm{cm}$ petri dish. Phage buffer $(10 \mathrm{mM}$ Tris- $\mathrm{HCl}[\mathrm{pH} 7.5], 1 \mathrm{mM}$ $\mathrm{MgCl}_{2}, 0.1 \mathrm{M} \mathrm{NaCl}$, and $10 \mathrm{mg}$ gelatin per liter) was used for the phage dilutions. After $5 \mathrm{~h}$ of incubation at $37^{\circ} \mathrm{C}$, the petri dish was moved to an $18 \times 24 \times 24$-in Plexiglas box, which served as an incubator to carry out long-term image acquisition. The incubation temperature was controlled to $37 \pm 0.2^{\circ} \mathrm{C}$ by heating the atmosphere inside the incubator with a heat blower (Masterflow, PGC Scientifics, Gaithersburg, MD). A proportional temperature controller (Digi-Sense, Cole-Parmer Instrument Co., Niles, IL) employed a thermistor probe (YSI Series 700, Cole-Parmer) inserted beside the sample and it accordingly adjusted the heat output. A variablevoltage controller (Cole-Parmer) independently regulated the flow rate of hot air. Sponges saturated with distilled water in recycled pipette-tip dispenser boxes were placed on the floor of the incubator to minimize evaporation from the agar.

Digital image acquisition. The petri dish was visualized using a chargecoupled device (CCD) camera (Sony XC-711, Industrial Vision Source) connected with an eye visual aid lens (Industrial Vision Source). The camera was mounted on a copy stand (Kaiser Fototechnik, CRG Precision Electronics) that readily adjusted for focusing. To enhance the contrast between the plaques and the host lawn, a circular fluorescent lamp surrounding the petri dish was used as the light source. The composite video signal from the CCD camera was fed to a computer (Power Macintosh 8100/80) for digitization. Digitized images with 256 gray levels $(0-255)$ were acquired with a frame grabber (Scion LG-3, Scion Corporation) of resolution $640 \times 480$ pixels. To continuously monitor the sample and follow the time history of plaque growth, a dark-field series of digital images were automatically acquired at fixed time intervals (every hour in our experiments) and stored on 1.2 GB rewritable optical cartridges driven by an external optical disk drive (Magic, MacProducts/Third Wave Sales) for processing.

Image processing and morphological characterization. The publicdomain NIH Image program (Wayne Rasband, National Institutes of Health) was used to process and analyze the digitized images. We applied the built-in macro programming language and wrote our own computer code to automate the acquisition and analysis. A new look-up table (LUT) function was generated by performing a linear histogram stretch of the acquired digital image to enhance the low contrast. The enhanced image was then convolved with a $7 \times 7$ mean filter to smooth out sharp noise ${ }^{31}$. The spatially filtered image was finally segmented to identify individual plaques by selecting a threshold that separated the background pixels from the pixels for a plaque. After identifying individual plaques, the area $A$ and perimeter $P$ were measured. The area equivalent radius $R_{e}$ of a plaque is defined as $R_{c}=(A / \pi)^{i / 2}$. The average propagation velocity was found by a linear regression to be the slope of $R_{e}$ versus time. Using a simple geometric measure based on the plaque area $A$ and perimeter $P$, we defined a parameter to characterize the population heterogeneity $(P H)$ :

$$
P H=\frac{P^{2}}{4 \pi A}-1
$$

which has a minimum of zero for a perfectly circular plaque, and approaches infinity as the shape approaches a line.

\section{Acknowledgments}

We are grateful for support provided by Research Initiation and Young Investigator Awards (to JY) from the National Science Foundation, the Kimball Foundation, and the Thayer School of Engineering at Dartmouth College.

1. Debyser, Z., De Vreese, K., Knops-Gerrits, P. P., Baekelandt, V., Bhikhabhai, R., Strandberg, B., et al. 1993. Kinetics of different human immunodeficiency virus type 1 reverse transcriptases resistant to human immunodeficiency virus type 1 specific reverse transcriptase inhibitors. Mol. Pharmacol. 43:521-526.

2. Domingo, E. 1989. RNA virus evolution and the control of viral disease. Prog Drug Res. 33:93-133.

3. Ecomoto, N., Sato, C., Kurosaki, M., and Marumo, F. 1994. Hepatitis C virus after interferon treatment has the variation in the hypervariable region of envelope 2 gene. J. Hepatol. 20:252-261.

4. el-Farrash, M. A., Kuroda, M. J., Kitazaki, T., Masuda, T., Kato, K., Hatanaka, M., et al. 1994. Generation and characterization of a human immunodeficiency virus type 1 (HIV-1) mutant resistant to an HIV-1 protease inhibitor. J. Virol. 68:233-239

5. Heinz, B. A., Rueckert, R. R., Shepard, D. A., Dutko, F. J., McKinlay, M. A. Fancher, M., et al. 1989. Genetic and molecular analysis of spontaneous mutants of human rhinovirus 14 that are resistant to an antiviral compound. $J$. Virol. 63:2476-2485.

6. Jacobsen, H. Yasargil, K. Winslow, D. L., Craig, J. C., Krohn, A Duncan, I. B., et al. 1995. Characterization of human immunodeficiency virus type 1 mutants with decreased sensitivity to proteinase inhibitor Ro 31-8959. Virol 206:527-534.

7. Lisziewicz, J., Sun, D., Klotman, M., Agrawal, S., Zamecnik, P., and Gallo, R. 1992. Specific inhibition of human immunodeficiency virus type 1 replication by antisense oligonucleotides: an in vitro model for treatment. Proc. Natl. Acad. Sci. USA 89:11209-11213.

8. Merigan, T. C. and Kundu, S. K. 1994. Human immunodeficiency virus envelope glycoproteins. J. AIDS [7 Suppl.] 1:S14-20.

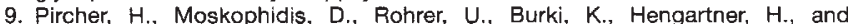
Zinkernagel, R. M. 1990. Viral escape by selection of cytotoxic T cell-resistant virus variants in vivo. Nature 346:629-633.

10. Smerdon, S. J., Jager, J., Wang, J., Kohlstaedt, L. A., Chirino, A. J., Friedman J. M., et al. 1994. Structure of the binding site for nonnucleoside inhibitors of the reverse transcriptase of human immunodeficiency virus type 1. Proc. Natl. Acad. Sci. USA 91:3911-3915.

11. Buonagurio, D. A., Nakada, S., Parvin, J. D., Krystal, M., Palese, P., and Fitch, W. M. 1986. Evolution of human influenza A viruses over 50 years: rapid, uniform rate of change in NS gene. Science 232:980-982

12. Cane, P. A. and Pringle, C. R. 1995. Evolution of subgroup A respiratory syncytial virus: evidence for progressive accumulation of amino acid changes in the attachment protein. J. Virol. 69:2918-2925

13. Goodenow, M., Huet, T., Saurin, W., Kwok, S., Sninsky, J., and Wain-Hobson, S. 1989. HIV-1 isolates are rapidly evolving quasispecies: evidence for viral mixtures and preferred nucleotide substitutions. J. AIDS 2:344-352.

14. Linnemann, C. C. J., Biron, K. K., Hoppenjans, W. G., and Solinger, A. M. 1990. Emergence of acyclovir-resistant varicella zoster virus in an AIDS patient on prolonged acyclovir therapy. AIDS 4:577-579.

15. Buynak, E. B. and Hilleman, M. R. 1966. Live attenuated mumps virus vaccine 1. Vaccine development. Proc. Soc. Exp. Biol. Med. 123:768-775

16. Clarke, D. K., Duarte, E. A., Moya, A., Elena, S. F., Domingo, E., and Holland, J. 1993. Genetic bottlenecks and population passages cause profound fitness differences in RNA viruses. J. Virol. 67:222-228.

17. Domingo, E., Sabo, D., Taniguchi, T., and Weissmann, C. 1978. Nucleotide sequence heterogeneity of an RNA phage population. Cell 13:735-744.

18. Kong, D. and Yin, J. 1995. Whole-virus vaccine development by continuous culture on a complementing host. Bio/Technology 13:583-586.

19. Larder, B. A., Coates, K. E., and Kemp, S. D. 1991. Zidovudine-resistant human immunodeficiency virus selected by passage in cell culture. J. Virol 65:5232-5236.

20. Durbin, R., Kane, A., and Stollar, V. 1991. A mutant of Sindbis virus with altered plaque morphology and a decreased ratio of 26 S:49 S RNA synthesis in mosquito cells. Virol. 183:306-312.

21. Palca, J. 1992. Errant HIV strain renders test virus stock useless. Science 256:1387-1388

22. Tikhonenko, A. T. and Linial, M. L. 1993. Transforming variants of the avian myc-containing retrovirus $\mathrm{FH} 3$ arise prior to phenotypic selection. $J$. Virol. 67:3635-3638.

23. Bauer, G. J., McCaskill, J. S., and Otten, H. 1989. Traveling waves of in vitro evolving RNA. Proc. Natl. Acad. Sci. USA 86:7937-7941.

24. McCaskill, J. S, and Bauer, G. J. 1993. Images of evolution: origin of spontaneous RNA replication waves. Proc. Natl. Acad. Sci. USA 90:4191-4195.

25. Yin, J. 1993. Evolution of bacteriophage T7 in a growing plaque. J. Bacteriol. 175:1272-1277.

26. Yin, J. 1991. A quantifiable phenotype of viral propagation. Biochem. Biophys. Res. Comm. 174:1009-1014.

27. Yin, J. and McCaskill, J. S. 1992. Replication of viruses in a growing plaque: a reaction-diffusion model. Biophys. J. 61:1540-1549.

28. Adams, M. H. 1959. Bacteriophages. Interscience Publishers, New York

29. Miller, J. H. 1972. Experiments in Molecular Genetics. Cold Spring Harbor Laboratory, Cold Spring Harbor, N.Y.

30. Studier, F. W. 1969. The genetics and physiology of bacteriophage T7. Virol. 39:562-574

31. Gonzalez, R. C. and Woods, R. E. 1992. Digital Image Processing. AddisonWesley Publishing Company, Inc., New York. 